\title{
The structure and rheology of molten ultra-high-molecular-mass polyethylene
}

\author{
P. Gao \\ Department of Chemical Engineering, The Hong Kong University of Science and Technology, \\ Clear Water Bay, Kowloon, Hong Kong
}

and M. R. Mackley*

Department of Chemical Engineering, University of Cambridge, Pembroke Street, Cambridge CB2 3RA, UK

(Received 17 January 1994; revised 14 April 1994)

\begin{abstract}
We report optical scattering and birefringence observations on molten ultra-high-molecular-mass polyethylene (UHMMPE) and correlate these observations with a structure in the melt relating to the original reactor powder. In the quiescent state, pressure annealing can cause the observed light scattering to disappear; however, subsequent flow of the material causes the scattering to develop again. A simple microrheological model is proposed to explain some of the structure, pressure annealing and flow effects that have been observed.
\end{abstract}

(Keywords: UHMMPE; melt structure; pressure annealing)

\section{INTRODUCTION}

It is normally assumed that molten polymers such as polyethylene are structure-free. The chains are considered to be in a random configuration and the interpenetrating polymer chains form a molten continuum (see, for example, refs 1 and 2). This lack of structure over a wide range of length scales results in most molten polymers being optically transparent and this property, for example, enables flow birefringence studies to be made without the complication of additional light-scattering effects. Work by $\mathrm{Han}^{3}$ and Wales ${ }^{4}$ shows a number of examples where polymers with molecular masses in the typical melt-processing regime show birefringence in the flowing state and no additional melt structure. More recent work by Checker et al..$^{5}$ and Aldhouse et al..$^{6}$ show additional flow birefringence data for normal processinggrade polyethylenes, where the values of $\bar{M}_{\mathrm{n}}$ and $\bar{M}_{\mathrm{w}}$ are of the order $10^{4}$ and $10^{5}$, respectively. Extensive rheological data on normal processing grades of polyethylene are also available in the literature (see, for example refs 7 and 8 ).

As the molecular mass of polyethylene is increased, processing becomes progressively more difficult and the literature on processing of ultra-high-molecular-mass polyethylene, UHMMPE, also becomes sparse. Hoechst ${ }^{9}$ describe conditions under which their UHMMPE Hostalen GUR can be processed. It is currently normal practice to either ram extrude molten UHMMPE, or under certain circumstances to use twin screw extruders. The transition between UHMMPE and normal processing-grade polyethylene is not precise; however, it has been shown ${ }^{10-12}$ that in the region $\overline{M_{\mathrm{w}}} \approx 500000$

* To whom correspondence should be addressed significant transitions occur in both processing and properties.

It has been known for many years that UHMMPE offers substantial advantages over normal processinggrade molecular mass polyethylenes in terms of toughness, fracture and wear improvement (see, for example, ref. 9). More recently it has also been shown that UHMMPE can be processed in a manner that can result in the manufacture of high modulus and strength structures ${ }^{13,14}$. The way in which molten UHMMPE can be processed is central to most aspects of property enhancement of this material and this paper relates specifically to a structure feature of UHMMPE melts that does not appear to be present in normal processing grades of polyethylene. Our objective is to demonstrate the existence of melt structure in UHMMPE and develop a model that is capable of some semiquantitative prediction of the effect. Previous observations on flow-induced crystallization in polyethylene melts at temperatures near the melting point have been made by one of the authors ${ }^{15}$. The effect described in this paper is different from the previously observed flow-induced crystallization effects. In the case described in this paper the origin of the structure relates to a memory of the initial particulate form of the material.

\section{EXPERIMENTAL}

\section{Materials}

Two different molecular mass grades of polyethylene materials were used in this study: BP Rigidex HM5411 $\left(\overline{M_{\mathrm{w}}}=2.5 \times 10^{5}, \overline{M_{\mathrm{n}}}=2.0 \times 10^{4} \mathrm{~kg} \mathrm{kmol}^{-1}\right)$ and Hifax $1900\left(\overline{M_{\mathrm{w}}}>10^{6}, \overline{M_{\mathrm{w}}}>10^{5} \mathrm{~kg} \mathrm{kmol}^{-1}\right)$. The former is available to us in the form of granules and the latter in 
the form of ex-reactor powders with an average particle size of $250 \mu \mathrm{m}$.

\section{Experimental set-up}

Optical observations were made using a flow cell developed in Cambridge and shown schematically in Figure 1. The flow cell was connected to a piston-driven Davenport ram extruder rheometer; the optical set-up and die geometries used in this study are similar to those described by Checker et al. ${ }^{5}$. The observation on the conventional polyethylene melt, Rigidex HM5411, was made using a die $1 \mathrm{~mm}$ wide $\times 1.5 \mathrm{~mm}$ deep, and, in the case of Hifax 1900, observations were made using a die $9 \mathrm{~mm}$ wide $\times 1.5 \mathrm{~mm}$ deep. The choice of die geometry dimensions was based on a number of factors. It will be shown that the UHMMPE exhibits significant light scattering and consequently there was a severe limitation on the depth of melt where light transmission was possible and $1.5 \mathrm{~mm}$ was found to be near to the maximum depth where transmission could be obtained. Given this depth the overall pressure drop and flow rate within the die were dependent on the width of the slit. In the case of UHMMPE a width of $9 \mathrm{~mm}$ was necessary in order to keep the overall pressure below the maximum of 200 bar. In the case of the low-molecular-mass polymer the pressure drops were considerably lower, but in order to observe any optical retardation fringes, a high flow rate was required through the slit. In order to achieve this a slit $1 \mathrm{~mm}$ wide was used as opposed to the $9 \mathrm{~mm}$ slit which produced no detectable fringes for the available flow rates. The observations were carried out by first

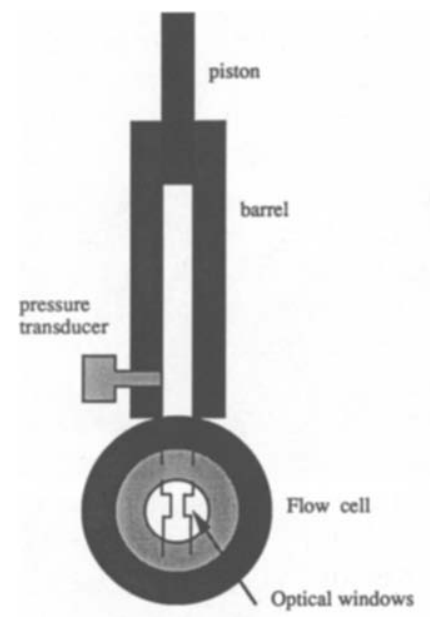

(a)

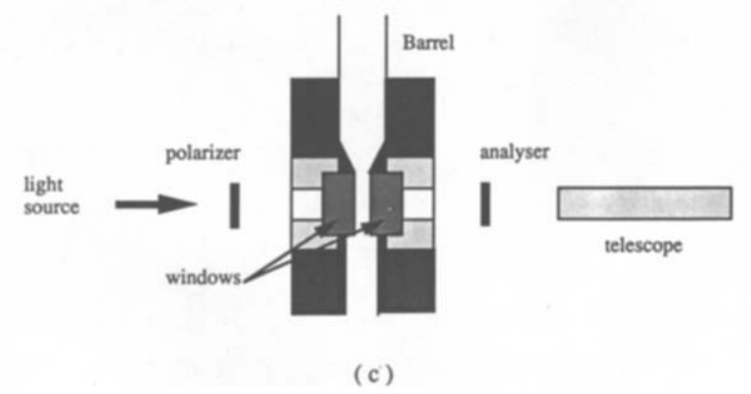

Figure 1 Schematic diagram showing the optical set-up. (a) Front elevation of flow cell; (b) schematic of the slit die used for observation; (c) side elevation of the optical arrangement

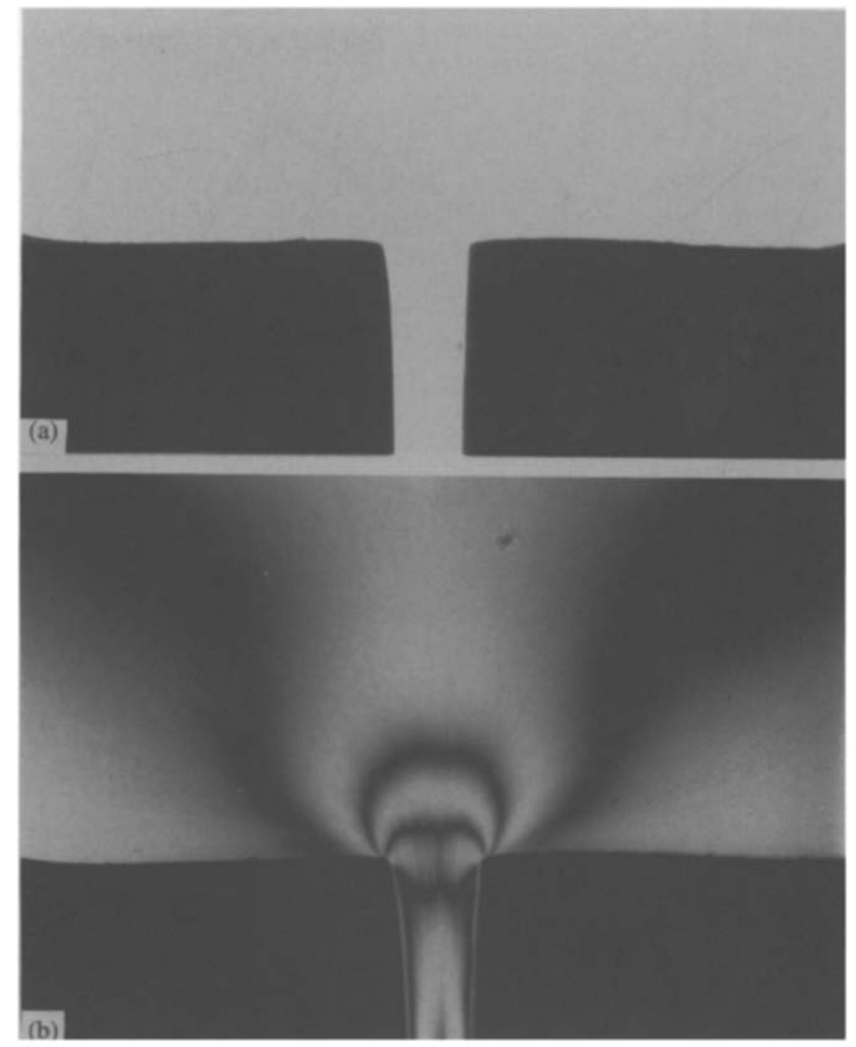

Figure 2 Photographs showing the flow behaviour of 'normal' $M_{\mathrm{w}}$ grade polyethylene Rigidex HM5411 flowing through a $1 \mathrm{~mm}$ width channel at flow rate $=134 \mathrm{~mm}^{3} \mathrm{~s}^{-1}, T=180^{\circ} \mathrm{C}, \Delta P \cong 50$ bar. (a) Bright field; (b) between crossed polarizers, polars $45^{\circ}$ to vertical

charging the material into the barrel at an elevated temperature of $180^{\circ} \mathrm{C}$ and annealing the material for $10 \mathrm{~min}$ before commencing extrusion and flow observations.

\section{Results}

The case of molten Rigidex polyethylene flowing into a slit is shown in Figure $2 a$, where the field of view shows no scattering when viewed in bright field and consequently we conclude that there is no structure within the melt at light wavelength resolution. In Figure $2 b$ classic flow birefringence is seen between crossed polaroids where the dark bands correspond to integer wavelength retardation of the two plane polarized beams that pass through the optically anisotropic flowing melt (see, for example, Checker et al. ${ }^{5}$ ). Both sets of observations confirm that this grade of polyethylene is free from structure both in the static and flowing melt. Observations in the $9 \times 1.5 \mathrm{~mm}$ die also showed no structure within the melt and in this case no fringes, because the overall retardation was too small to detect.

In contrast to the previous observations, UHMMPE shows striking differences. Figure 3 is a representative photograph of UHMMPE flowing within a $9 \times 1.5 \mathrm{~mm}$ channel. The channel width had to be increased from the $1 \mathrm{~mm}$ width used in Figure 2 in order to facilitate any extrusion of the UHMMPE below the pressure limit of the rheometer (200 bar). Figure 3 was taken using crossed polarized light; however, because of the intense scattering within the material the observations are essentially the same in both polarized and bright field. 
Three distinct features can be seen in Figure 3. First, there is intense scattering within the flowing melt; this gives rise to a granular texture of the melt which we believe is associated with the residue memory of the initial reactor particles. This region is most clearly seen in the top right-hand corner of the photo. Secondly, during flow, black regions can be seen within the field of view

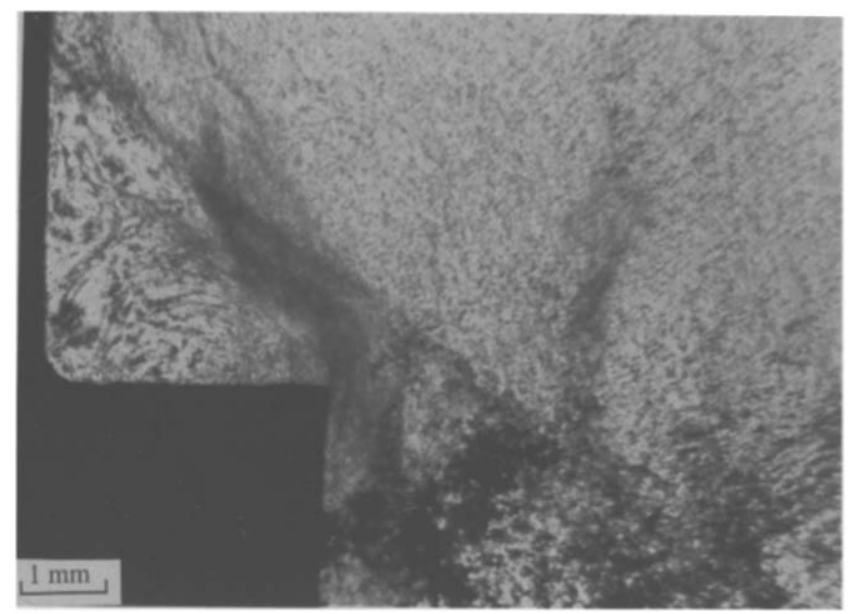

Figure 3 Photograph showing the flow behaviour of UHMMPE Hifax 1900 flowing through a $9 \mathrm{~mm}$ width channel at flow rate $=13.4 \mathrm{~mm}^{3} \mathrm{~s}^{-1} ; T=180^{\circ} \mathrm{C}, \Delta P \cong 150 \mathrm{bar}$ of the flowing melt. These appear at the bottom of this photograph. We believe that these black regions are either voids that penetrate through the whole melt, or that there is a glass/air/polymer interface at one or both of the glass walls which is often associated with melt fracture. In either case, we believe that the optically observed dark contrast can result from refraction of the plane-propagated light at a curved surface (or nonuniform interface). This could well be associated with a region of stick/slip at the wall. Visual observations recorded using a video camera during the extrusion process clearly indicate that the material shows an initial stick/slip behaviour followed by a definite slip component at the wall. Simultaneous pressure drop measurements also show an unstable fluctuating pressure profile for these extrusion conditions. Thirdly, if the extrusion has continued for a number of minutes we note that the optical contrast in the near-stagnant corner region (on the left-hand side of the photograph) of the die is different from the material flowing within the channel. In the corner of the die the intensity of the light scattering reduces with time, to a level sufficient to enable birefringence to be detected. A sharp boundary develops between the near-stagnant birefringent material and the strongly scattering flowing polymer.

Figures $4 a-d$ show the structure evolution on cessation of flow as a function of time under a high residue pressure of 100 bar. Figure $4 a$ shows the instant at which the flow
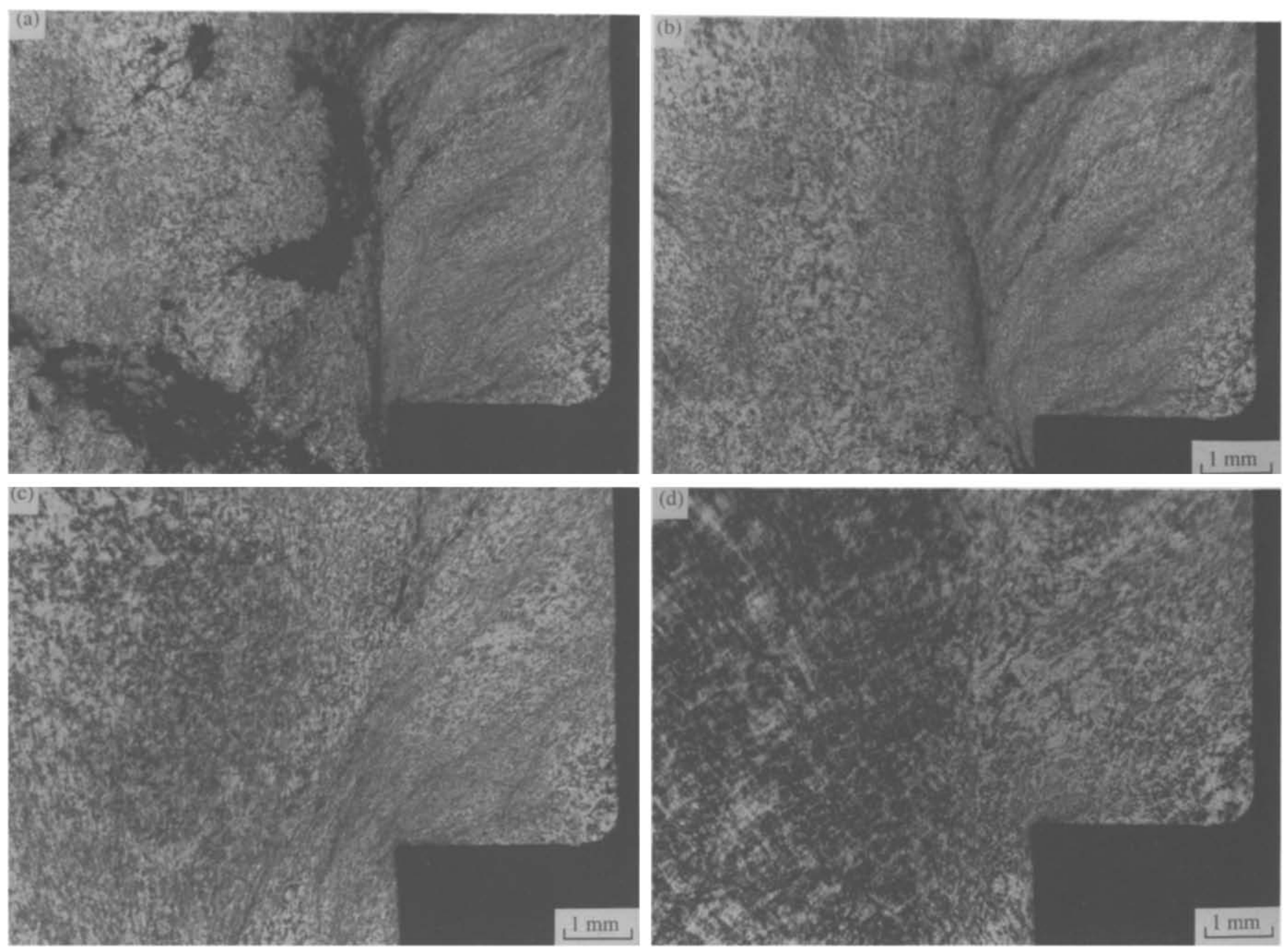

Figure 4 Photographs showing the effect of pressure annealing on the structure of UHMMPE at $180^{\circ} \mathrm{C}$ and an annealing pressure of 100 bar for material flowing through a channel width of $9 \mathrm{~mm}$ at an initial flow rate of $13.4 \mathrm{~mm}^{3} \mathrm{~s}^{-1}$ prior to stoppage. (a) $t=0 \mathrm{~s}$; (b) $t=30 \mathrm{~s}$; (c) $t=5 \mathrm{~min}$; (d) $t=1 \mathrm{~h}$. Polars crossed at $45^{\circ}$ to the vertical 
was just stopped at an initial flow rate of $13.4 \mathrm{~mm}^{3} \mathrm{~s}^{-1}$ at $180^{\circ} \mathrm{C}$. In this case flow structure and dark regions can still be seen. When the piston is stopped, the pressure is generally maintained without any further significant flow and Figures $4 b-d$ show the time evolution of events for pressure annealing at $100 \mathrm{bar}$ and $T=180^{\circ} \mathrm{C}$. Initially, after approximately $30 \mathrm{~s}$ the black regions within the melt disappear. We believe the most likely explanation for this is that the polymer flows into a glass/air/polymer gap at the wall and thereby allows the light to propagate through the material as elsewhere in the die. With further pressure annealing, which might be of the order of $1 \mathrm{~h}$, the scattering within the melt diminishes and if the material is viewed between crossed polarizer the 'frozen in' flow stress birefringence fringe pattern is observed, as shown in Figure 4d. These observations show that the time constant for structure removal (within the wavelength of light) is of the order of $1 \mathrm{~h}$ under these conditions. It should be emphasized that thermal degradation under high pressures of $100 \mathrm{bar}$ appears to be minimal since the extrudate does not appear to change in colour within the period of $3 \mathrm{~h}$. At a pressure of the order of less than about 10 bar, pressure annealing appeared to have no effect on structure removal.

If in the case of high-pressure annealing, where the structure is effectively removed, flow is then recommended, a time evolution sequence, as shown in Figure $5 a$ to $d$, is observed. In this case the initial structure-free, but birefringent, material seen in Figure $5 a$ transforms and reverts with the initiation of flow to its former structured melt. This implies that although pressure annealing can apparently remove the structure, the material could, however, retain a memory of the structure which can manifest itself again during subsequent flow. The observations reported here are quite distinct from flow-induced crystallization effects as seen in lowmolecular-mass polymers that have been reported previously by one of the authors (Mackley et al. ${ }^{15}$ ).

\section{Rheological measurement}

Measurements were made on both the extrusion pressure profiles and linear viscoelastic behaviour of the molten polymer. In Figure 6 the pressure-time curve is shown for Hifax 1900 flowing at $13.4 \mathrm{~mm}^{3} \mathrm{~s}^{-1}$ at $180^{\circ} \mathrm{C}$ through the $9 \times 1.5 \mathrm{~mm}$ channels. The broken line is for polymer which has been pressure annealed. In this case, with the onset of extrusion the pressure passes through a clear pressure overshoot of the order of 190 bar and then an erratic extrusion follows where a stick/slip motion is observed for the extrudate. When this occurs the pressure profile also oscillates about a mean value of the order of 140 bar. If pressure annealing had not taken place the subsequent flow response was different and this is shown as a solid line in Figure 6. For this case no pressure overshoot is observed; however, when flow is fully established the extrusion characteristics are very
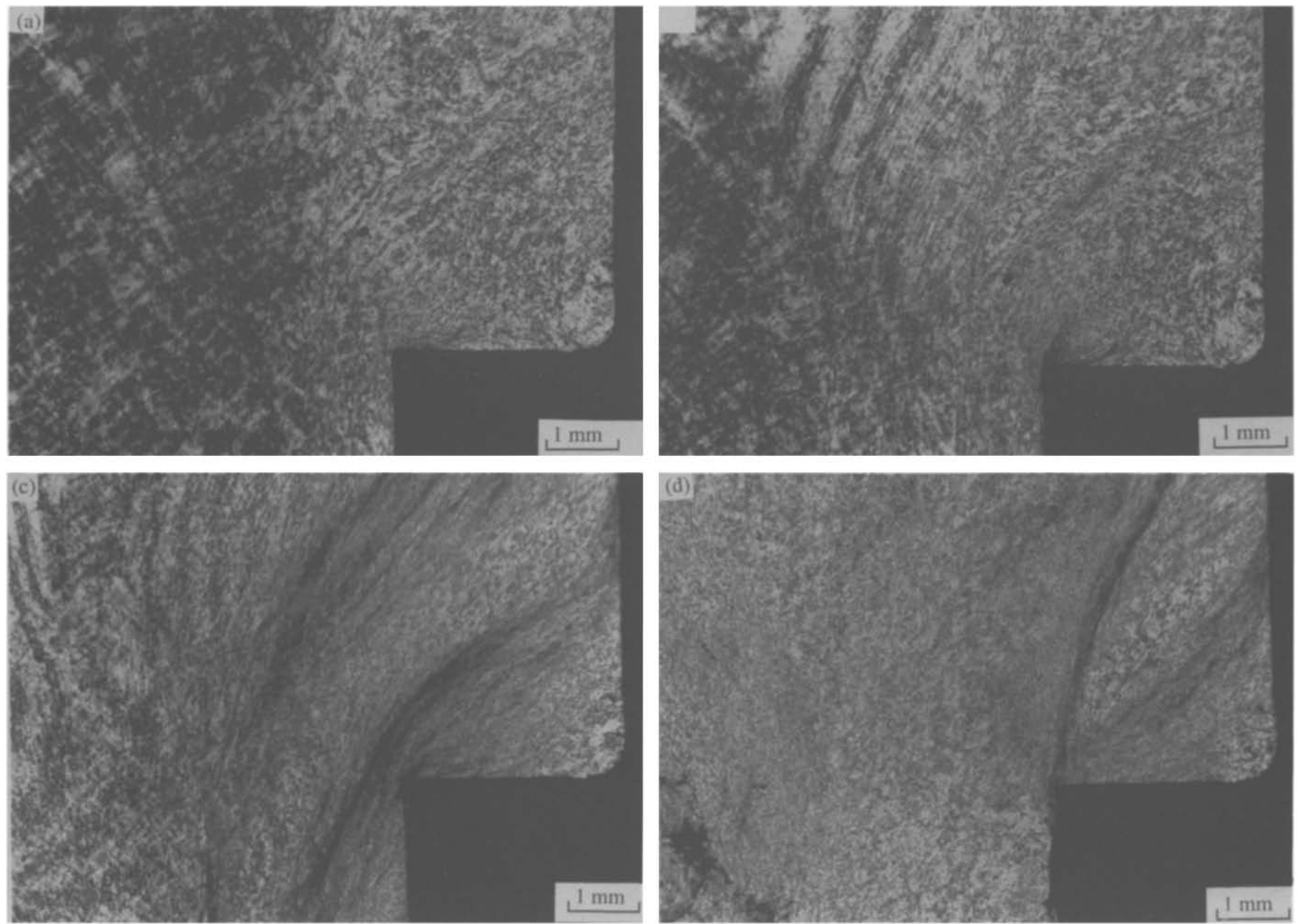

Figure 5 Photographs showing the effect of flow on the annealed structure after an initial annealing temperature of $180^{\circ} \mathrm{C}$ and pressure of 100 bar for $1 \mathrm{~h}$. (a) $t=0 \mathrm{~s}$; (b) $t=5 \mathrm{~s}$; (c) $t=7 \mathrm{~s}$; (d) $t=15 \mathrm{~s}$. Flow rate $=13.4 \mathrm{~mm}^{3} \mathrm{~s}^{-1}$. Polars crossed at $45^{\circ}$ to the vertical 


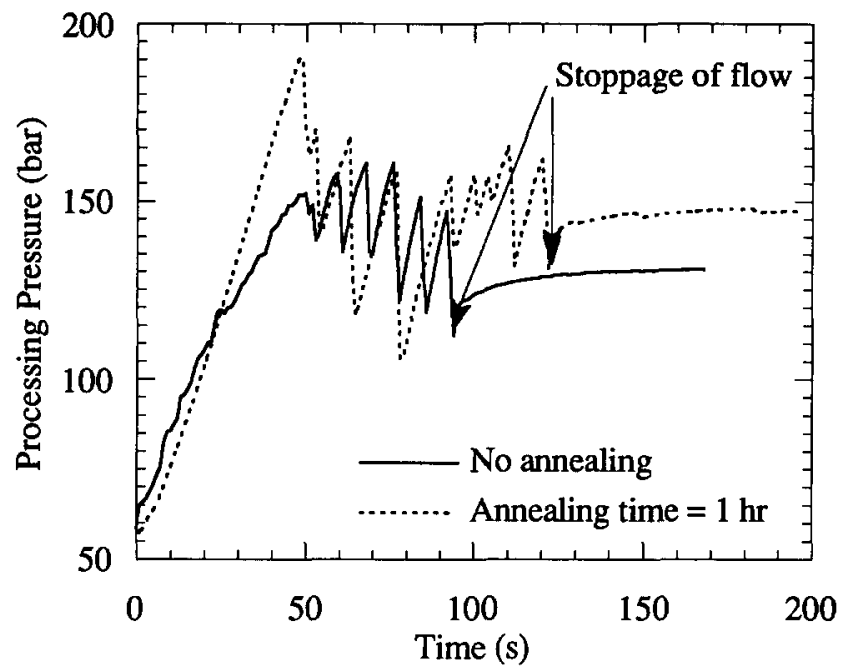

Figure 6 Processing pressure drop versus time curves for annealed and unannealed UHMMPE Hifax 1900 at a flow rate of $13.4 \mathrm{~mm}^{3} \mathrm{~s}^{-1}$ and a temperature of $180^{\circ} \mathrm{C}$. The initial annealing pressure $=100 \mathrm{bar}$, $T=180^{\circ} \mathrm{C}$

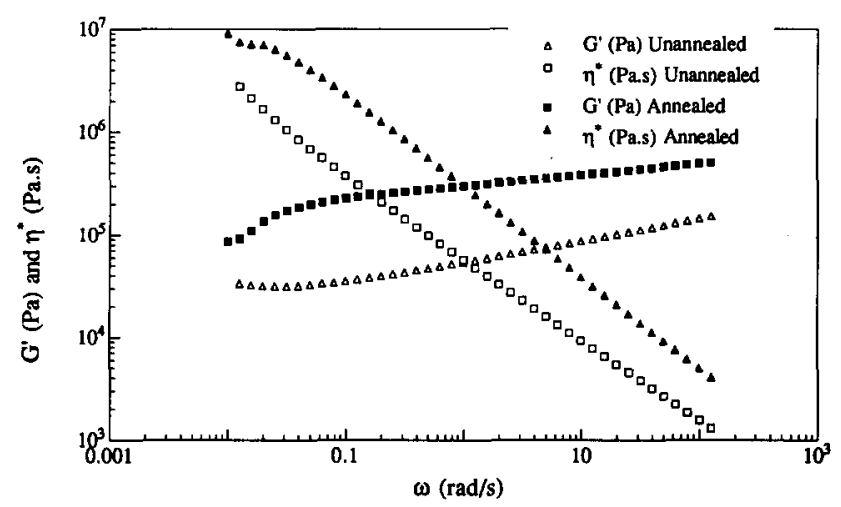

Figure 7 Small strain frequency sweeps for structured and annealed UHMMPE Hifax 1900 melts at $180^{\circ} \mathrm{C}$; gap $=1 \mathrm{~mm}$; strain $=3 \%$. The initial annealing times for the structured and annealed melts are $5 \mathrm{~min}$ and $1 \mathrm{~h}$, respectively, at an annealing pressure of $100 \mathrm{bar}$ and a temperature of $180^{\circ} \mathrm{C}$

similar. In both cases, on the cessation of piston movement the pressure within the melt surprisingly remained near the value at flow cessation. No significant stress relaxation occurred on the time-scale of the order of $1 \mathrm{~h}$.

Small strain dynamic rate sweep experiments were also carried out on both the structured and pressure-annealed ultra-high-molecular-mass polyethylene Hifax melts. Both samples were prepared using a hydraulic press at a temperature of $180^{\circ} \mathrm{C}$ and a pressure of 100 bar. They differ in annealing time. The structured melt was prepared at an annealing time of $5 \mathrm{~min}$ and the latter after annealing of $1 \mathrm{~h}$. The dynamic rate sweep experiments were performed using an RDS II Rheometrics at $180^{\circ} \mathrm{C}$ within the linear viscoelastic region of the material. Detailed descriptions on the experimental set-up and sample geometries have been given elsewhere ${ }^{16}$. Data are shown in Figure 7 for both the structured and structure-free melt at a maximum strain of $3 \%$. In both cases the storage modulus dominates the loss modulus over the entire frequency range tested; however, the annealed melt shows a substantially higher $G^{\prime}$ and $\eta^{*}$ value than the structured melt. This suggests that the presence or absence of melt structure can also substantially affect melt rheology.

\section{MODELLING}

The light-scattering contrast we observe occurs because there are regions of strong refractive index gradients within the material that are presumably caused by the imperfect matching of the reactor powder grain boundaries. The pressure annealing process in particular suggests to us that two factors are involved in the formation of a melt continuum. If the material starts as a powder, a necessary but not sufficient condition to form a full melt is that the adjacent faces of reactor powders must be in intimate physical contact with each other. When the faces are in contact, in order to form a full melt it is then necessary for polymer chains to interdiffuse across this boundary in order that the density of polymer crossing the boundary is the same as that in any arbitrary plane within the melt. The physical contact of the interfaces will be controlled mainly by the pressure within the melt and the viscosity of the molten polymer. Diffusion across the boundary will be controlled by reptation mechanisms.

In order to model this process we consider a very simplified case. Assume the interface between grains is of the form shown schematically in Figure 8 . The situation is idealized in representing one surface as having a cavity of diameter $D$ and depth $\Delta L$. The other surface is assumed to constitute a high-viscosity melt interface.

If we assume that the material behaves as a power law fluid, the volumetric flow rate of polymer flowing into a capillary of diameter $D$, length $\Delta L$ and differential pressure $P$ is given by ${ }^{17}$,

$$
Q=\frac{n}{(3 n+1)} \frac{\pi D^{3}}{8}\left(\frac{P D}{4 K \Delta L}\right)^{1 / n}
$$

where the shear stress $\sigma$ is given by $\sigma=K \dot{\gamma}^{n}, K$ and $n$ are the material constant and power law index, respectively, $\dot{\gamma}$ is the shear rate and $P$ is the differential pressure across
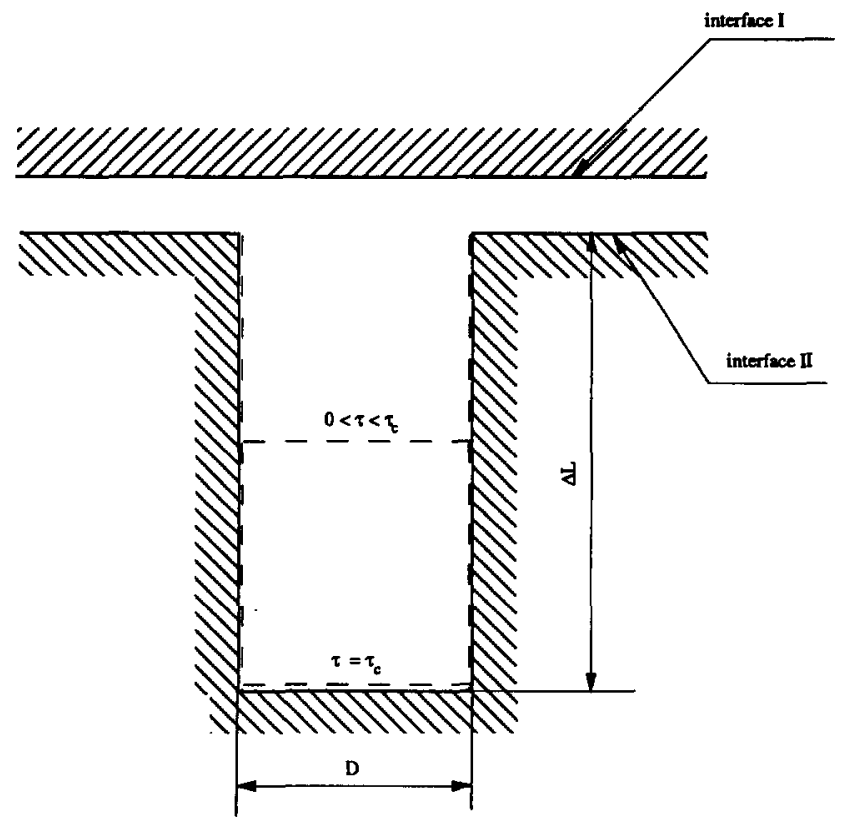

Figure 8 Schematic diagram showing the simplified two interfacial structures coming into contact 


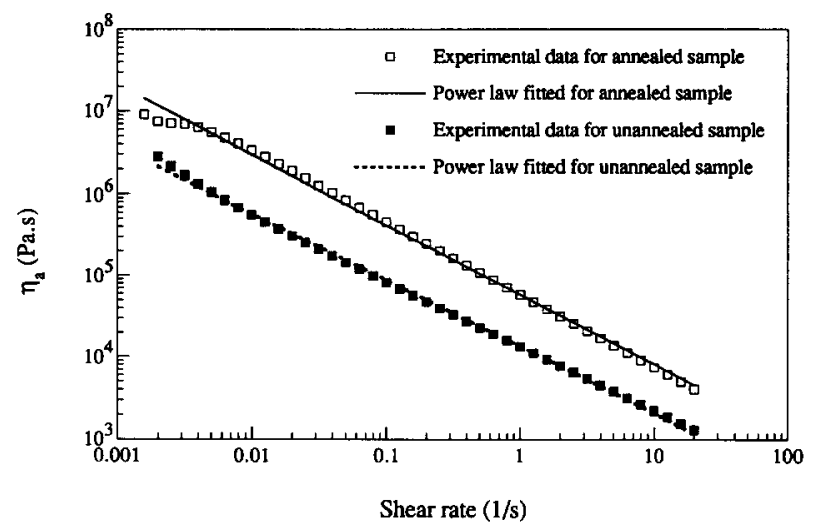

Figure 9 Apparent viscosity versus shear rate curve obtained from the complex viscosity data (Figure 7) using the Cox-Merz rule for the unannealed UHMMPE melt

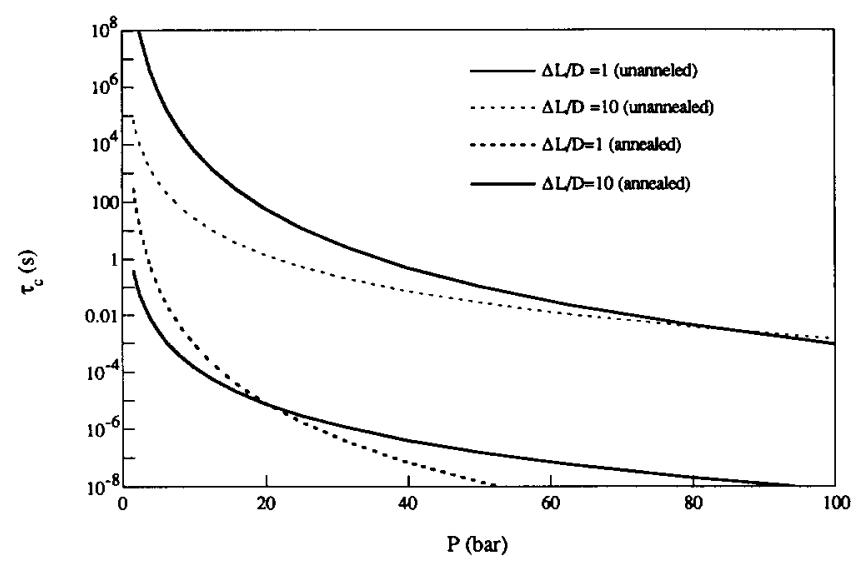

Figure 10 Effect of processing pressure and aspect ratio $\Delta L / D$ on the contact time $\tau_{\mathrm{c}}$ for UHMMPE Hifax 1900

the cavity. The time required to fill the cavity, $\tau_{c}$, is given by

$$
\tau_{\mathrm{c}}=V / Q=\pi D^{3} / 4 Q=\frac{2(3 n+1)}{n}\left(\frac{4 K}{\Delta P} \frac{\Delta L}{D}\right)^{1 / n}
$$

We assign the contact time for interfaces as $\tau_{c}$, and note that this is not only dependent on $\Delta L / D$ but also the magnitude of $P$ and the rheological properties of the melt. If we use the complex viscosity data of UHMMPE and further assuming the Cox-Merz rule, the complex viscosity data is equivalent to the apparent viscosity where $\eta^{*}(\omega)=\eta_{\mathrm{a}}(\dot{\gamma})$ when $\omega=\dot{\gamma}$. Using this relation the $\eta^{*}$ data are fitted to a power law relation and the best fit data yield $K=1.35 \times 10^{4} \mathrm{~Pa} \mathrm{~s} \mathrm{~s}^{n}$ and $n=0.19$ for the unannealed material as shown in Figure 9. These data correspond to a very high viscosity melt with strong shear thinning characteristics.

In Figure 10, we plot the contact time $\tau_{\mathrm{c}}$ for different values of $\Delta L / D$. The contact time is calculated using both unannealed and annealed rheological data. From these curves it can be seen that pressures in excess of $\sim 5$ bar are necessary to ensure contact within $\sim 100 \mathrm{~s}$. Clearly, increasing pressure decreases the interface contact time and increasing the $\Delta L / D$ ratio increases the contact time.

Once physical contact between interfaces has been established, chain diffusion can take place. We associate this characteristic time with the reptation time of the chain $\tau_{r}$ (ref. 2). It is now well established that $\tau_{r}$ varies

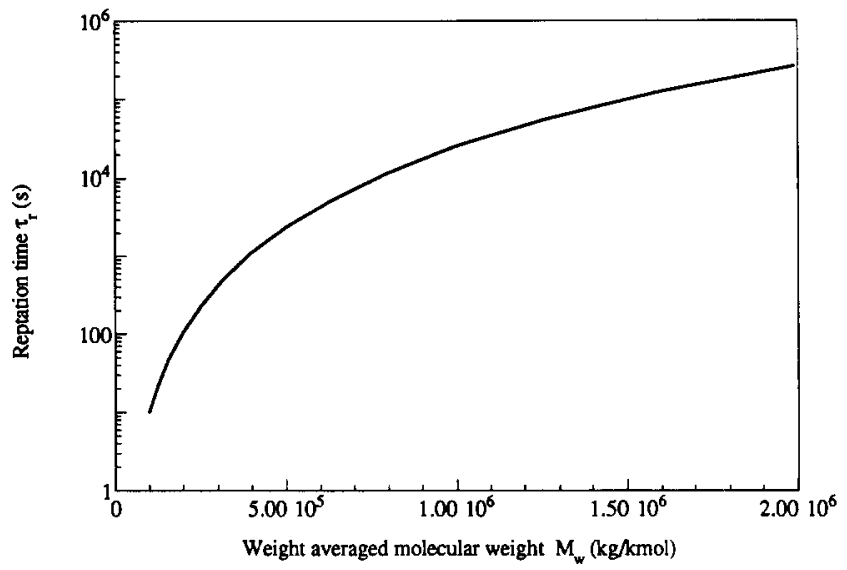

Figure 11 Effect of mass-averaged molecular mass on the particle interfacial diffusion time $\tau_{\mathrm{r}}$ for UHMMPE Hifax 1900

with weight-averaged molecular mass as

$$
\tau_{\mathrm{r}} \propto M_{\mathrm{w}}^{3.4}
$$

If the reptation time for a lower molecular mass polyethylene is known, the required reptation time can then be obtained. Therefore the overall time for a structure-free melt can then be estimated as

$$
\tau=\tau_{\mathrm{c}}+\tau_{\mathrm{r}}
$$

An example of the effect of molecular mass on the value of $\tau$ is given in Figure 11. The calculation of reptation time was based on a reference reptation time of $10 \mathrm{~s}$ for the molecular mass of $100000 \mathrm{~kg} \mathrm{kmol}^{-1}$ (ref. 18). The scale ranges from approximately $10 \mathrm{~s}$ to $10^{6} \mathrm{~s}$ for molecular mass increasing from 100000 to $2 \times 10^{6} \mathrm{~kg} \mathrm{kmol}^{-1}$. Even if annealing is allowed for $1 \mathrm{~h}$, polyethylenes of molecular mass $\geqslant 500000 \mathrm{~kg} \mathrm{kmol}^{-1}$ will not have had sufficient time to reptate fully.

From these data it is evident that elevated pressure can satisfy the contact condition but longer time is needed to satisfy the diffusion condition. From the approximate analysis, for example, a pressure of 40 bar will give a contact time of $10^{-7}-1 \mathrm{~s}$. An annealing time of $15 \mathrm{~min}$ will mean that only polymers with a molecular mass of less than about 200000 will be expected to have fully diffused across the boundary, but polymer with a $M_{w}$ greater than this value will remain within the domain of its original particulate form.

\section{DISCUSSION AND CONCLUSIONS}

The model we have proposed can explain qualitatively some of the results we have observed. From the simple calculations carried out it is clear that for UHMMPE both elevated pressures and long annealing times are necessary in order to remove completely the memory of interface within the melt. It would appear that the pressure annealing we have carried out is long enough for some interchain diffusion to occur but insufficient for the whole memory of the interface to be lost. When flow reoccurs after pressure annealing the high-molecularmass component in each reactor grain retains its memory and shear between each grain reoccurs with the reappearance of the particle boundaries.

The pressure annealing effect of UHMMPE could also explain a further observation. During the continuous extrusion of UHMMPE through the flow cell we noticed 
that in the near-stagnant region of the flow, upstream and to the side of the die throat, there was less light scattering than from material flowing into the die. A boundary developed between flowing scattering material and near-stagnant material in the corner of the die. This effect can be seen in Figure 3. Clearly, the residence time of material in the near-stagnant region is very much greater than for the flowing material and presumably this material has spent sufficient time in the die at high enough pressure for the melt interface structure to disappear.

It would appear from the observations we have made that the rheology of UHMMPE will be influenced by both the molecular mass distribution of the polymer and the nature of the interparticle structure created by the original reactor powder, and this conclusion is supported by the significant rheological change seen in the $G^{\prime}, G^{\prime \prime}$ data. Pressure annealing has caused the bulk material to develop a yield stress before flow occurs and a significant increase in $G^{\prime}$ and $\eta^{*}$ is observed. These complications will not exist in normal processing-grade polyethylenes because the contact time will be short due to the lower zero shear viscosity of the material and the reptation time of the material will also be less because of the lower molecular mass.

Clearly there are many gross assumptions to the model we have presented. In particular the exact geometry of the interparticle boundary will be very much more complex than we have assumed. In addition, both boundaries will be compliant rather than our assumption of a single viscous boundary flowing into a rigid pore. Elasticity may well have a significant effect on the rheology of the interface contact and reptation is only one of a number of mechanisms whereby polymer diffusion across a boundary can take place. We believe, however, that pressure-driven viscous flow is the dominant mechanism for interface contact and polymer reptation is certainly the controlling mechanism for the self-diffusion of high-molecular-mass chain. On this basis we would hope for first-order accuracy from the model.

Our observations suggest that a more homogeneous UHMMPE product is likely to be obtained by pressure-driven compression moulding where little flow is involved as compared to ram extrusion processing where significant shear occurs during the forming process. Our observations also show that wall slip does occur during extrusion processing of this material and this factor would need to be considered if the extrusion process were to be modelled.

In conclusion, normal processing-grade polyethylene melts have little difficulty in forming uniform melts; however, both the high viscosity and long reptation times associated with UHMMPE make it very difficult to envisage that under practical processing conditions it is possible to form a purely homogeneous polyethylene melt of this material. This factor should be borne in mind when considering both the melt rheology and also the final solid state properties of the material.

\section{ACKNOWLEDGEMENTS}

The authors thank DSM Research for their financial support during the course of the work.

\section{REFERENCES}

1 Flory, P. J. 'Principles of Polymer Chemistry', Cornell University Press, Ithaca, NY, 1968

2 Doi, M. and Edwards, S. F. 'The Theory of Polymer Dynamics', Oxford University Press, Oxford, 1986

3 Han, C. D. 'Rheology in Polymer Processing', Academic Press, New York, 1976

4 Wales, J. L. S. 'The Application of Flow Birefringence to Rheological Studies of Polymer Melts', Delft University Press, Delft, The Netherlands, 1976

5 Checker, N., Mackley, M. R. and Mead, D. W. Phil. Trans. $R$. Soc. London 1983, A308, 451

6 Aldhouse, S. T. E., Mackley, M. R. and Moore, I. P. T. J. Non-New'tonian Fluid Mech. 1986, 21, 359

7 Wagner, M. H. and Laun, H. M. Rheol. Acta 1978, 17, 138

8 Laun, H. M. Rheol. Acta 1978, 17, 1

9 Hoechst Plastics. 'Hostalen G High Density Polyethylene', Hoechst Plastics, 1969

10 Bashir, Z., Odell, J. A. and Keller, A. J. Mater. Sci., 1986, 21, 3993

11 Bashir, Z. and Keller, A. Colloid Polym. Sci. 1989, 267, 116

12 Waddon, A. J. and Keller, A. J. Polym. Sci., Polym. Phys. (B) 1992, 30, 923

13 Smith, P., Lemstra, P. J. and Booij, H. C. J. Mater. Sci. 1981, 15, 505

14 Barham, P. J. and Keller, A. J. Mater. Sci. 1985, 20, 2281

15 Mackley, M. R., Frank, F. C. and Keller, A. J. Mater. Sci. $1975,10,1501$

16 Gao, P. and Mackley, M. R. Polymer 1992, 19, 4075

17 Mackley, M. R. in 'The Physical Principles of Rheological Measurements' (Eds A. A. Collyer and D. W. Clegy), Elsevier, Amsterdam, 1988, Ch. 1

18 Kamath, V. M. and Mackley, M. R. J. Non-Newtonian Fluid Mech. 1989, 32, 119 